\title{
Stochastic Phenomena in One-Dimensional Rulkov Model of Neuronal Dynamics
}

\author{
Irina Bashkirtseva \\ Ural Federal University, Lenina 51, Ekaterinburg 620000, Russia \\ Correspondence should be addressed to Irina Bashkirtseva; irina.bashkirtseva@urfu.ru
}

Received 14 October 2014; Revised 19 March 2015; Accepted 20 March 2015

Academic Editor: Binggen Zhang

Copyright ( 2015 Irina Bashkirtseva. This is an open access article distributed under the Creative Commons Attribution License, which permits unrestricted use, distribution, and reproduction in any medium, provided the original work is properly cited.

\begin{abstract}
We study the nonlinear Rulkov map-based neuron model forced by random disturbances. For this model, an overview of the variety of stochastic regimes is given. For the parametric analysis of these regimes, the stochastic sensitivity functions technique is used. In a period-doubling zone, we analyze backward stochastic bifurcations modelling changes of modality of noisy neuron spiking. Noise-induced transitions in a zone of bistability are considered. It is shown how such random transitions can generate a new neuronal regime of the stochastic bursting and transfer the system from order to chaos. A transient zone of values of noise intensity corresponding to the onset of noise-induced bursting and chaotization is localized by the stochastic sensitivity functions technique.
\end{abstract}

\section{Introduction}

Since the pioneering work of Hodgkin and Huxley [1], neuron models attract attention of many researchers [2]. Continuoustime systems for modeling of the excitable neuron systems are widely studied. Various regimes such as spiking, bursting, fast-slow dynamics, and Canard oscillations are subject to the active investigation on the base of continuous-time models of Hindmarsh-Rose, FitzHugh-Nagumo, Morris-Lecar, and others.

Discrete-time dynamical neuron models began to be studied only recently $[3,4]$. Rulkov system [5] is one of the discrete models which adequately reflect main phenomenological features of neuronal dynamics. The simple twodimensional Rulkov map-based model exhibits three main types of neural activity, such as quiescence, tonic spiking, and bursting $[6,7]$. Note that it is impossible to model bursting in a framework of two-dimensional continuous-time systems.

Random noise is an inevitable attribute of any living system. In presence of stochastic disturbances, nonlinear system can exhibit unexpected regimes of dynamics which have no analogue in the deterministic case [8-12]. An analysis of noise-induced phenomena in neuron models is a challenging problem of the modern neurodynamics $[13,14]$.

Full description of the dynamics of the probabilistic distribution is given by Fokker-Planck-Kolmogorov equation
[15] for continuous-time systems and by Frobenius-Perron equation [16] for discrete systems. An analytical solution of these equations is possible only in very special cases. So, for the investigation of stochastic dynamics, a method of direct numerical simulation is widely used. This method is a time-consuming for the parametric analysis. Thus, a development of the asymptotic approximations is a highly relevant area of research. For the constructive analysis of the stochastic attractors of dynamical systems, a stochastic sensitivity functions technique was elaborated [17-19].

In the present paper, we consider one-dimensional Rulkov model of the neuronal dynamics [5]. Even in the deterministic case, this model possesses a wide variety of dynamical regimes and bifurcations, such as regular and chaotic attractors, multistability, bifurcations of perioddoubling, and crisis [3]. In particular, Rulkov model exhibits transitions from quiescence to spiking regime, both tonic (periodic) and chaotic. However, this deterministic system still cannot model the neuronal bursting. In what follows, we show how the neuronal bursting appears in presence of noise.

In current paper for in Rulkov model, we study stochastic deformations of the spiking regime and suggest a constructive method for parametric analysis of the noise-induced bursting.

In Section 2, after the short overview of the deterministic attractors and bifurcations, we present stochastic 


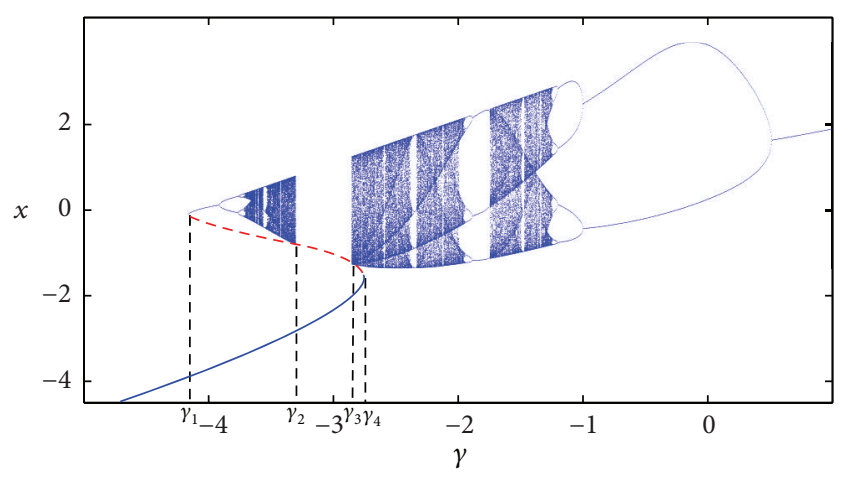

Figure 1: Attractors of the deterministic Rulkov model.

phenomena in this model. Along with quantitative changes of the random states' dispersion, increasing noise generates qualitative transformations of stochastic dynamics. In the stochastic Rulkov model, noise-induced transitions and stochastic bifurcations are observed. In particular, it is shown how random transitions between stable equilibrium and chaotic attractor cause noise-induced bursting.

For the parametric analysis of these phenomena, the stochastic sensitivity functions technique and confidence domains method are used in Section 3. Here, backward bifurcations of reducing of the multiplicity of stochastic cycles, and noise-induced bursting with transitions from order to chaos are studied.

\section{Stochastic Phenomena in Rulkov Model}

Consider a stochastic Rulkov system:

$$
x_{t+1}=\frac{\alpha}{1+x_{t}^{2}}+\gamma+\varepsilon \xi_{t},
$$

where $x$ is a membrane voltage in a neuron and $\gamma$ is a gatingion concentration. Random fluctuations in $\gamma$ are modelled by the uncorrelated Gaussian process $\xi_{t}$ with parameters $\mathrm{E}\left(\xi_{t}\right)=$ $0, \mathrm{E}\left(\xi_{t}^{2}\right)=1 ; \varepsilon$ is the noise intensity.

The deterministic system (1) (with $\varepsilon=0$ wherein) was introduced in [5] as a fast part of two-dimensional fast-slow system modeling bursting. In the present paper, we fix the parameter $\alpha=4.1$ and vary the parameter $\gamma$.

In Figure 1, attractors of the deterministic Rulkov model are shown for $-5<\gamma<1$. As one can see, this onedimensional simple model demonstrates a wide variety of dynamical regimes. Denote $\gamma_{1}=-4.162, \gamma_{2}=-3.3, \gamma_{3}=$ -2.849 , and $\gamma_{4}=-2.729$. In the interval $-5<\gamma<\gamma_{4}$, the system has a stable equilibrium $M_{1}$ (see lower curve in Figure 1). Points $\gamma_{1}$ and $\gamma_{4}$ mark the saddle-node bifurcations. When the parameter $\gamma$ passes $\gamma_{1}$ from the left to right, a stable equilibrium $M_{2}$ and unstable equilibrium $M_{3}$ are born. Unstable equilibrium $M_{3}$ is shown for $\gamma_{1}<\gamma<\gamma_{4}$ by red dashed line. At $\gamma=\gamma_{4}$, the equilibria $M_{1}$ and $M_{3}$ merge and annihilate.

For $\gamma_{1}<\gamma<\gamma_{2}$ (see upper part in Figure 1), the deterministic Rulkov model exhibits standard Feigenbaum's bifurcation "tree" with period doubling and intermittency of order and chaos. Similar tree of attractors is plotted in upper part of Figure 1 for $\gamma_{4}<\gamma<1$. These trees are separated by the interval $\left(\gamma_{2}, \gamma_{3}\right)$ with boundary points $\gamma_{2}$ and $\gamma_{3}$ which mark crises. System is monostable for $\left(-5, \gamma_{1}\right) \cup\left(\gamma_{2}, \gamma_{3}\right) \cup\left(\gamma_{4}, 1\right)$ and bistable for $\left(\gamma_{1}, \gamma_{2}\right) \cup\left(\gamma_{3}, \gamma_{4}\right)$.

So, the deterministic Rulkov map-based system (1) can model the following basic regimes of neuronal activity: quiescence (equilibrium), tonic spiking (cycles), and chaotic spiking (chaotic attractors). Consider further how noise affects these regimes of neuronal activity.

Under stochastic disturbances the solutions of system (1) leave the deterministic attractors and form some stationary probabilistic distributions around them. In Figure 2, random states $x_{t},(t=1001, \ldots, 1200)$ of system (1) solutions starting from $x_{0}=0$ are plotted for different values of the noise intensity. Weak noise slightly washes out the thin structure of deterministic attractors (see Figure 2(a) for $\varepsilon=0.003$ ). As noise intensity increases, a dispersion of random states grows. Along with quantitative changes of the dispersion, increasing noise implies qualitative transformations of stochastic dynamics.

First, branches of multiple cycles merge. For example, for $\varepsilon=0.01$, the only stochastic 2 cycles can be seen (Figure $2(\mathrm{~b})$ ). In Figure 2(c), for $\varepsilon=0.04$, the upper attractor looks like the stochastic equilibrium.

Second, in the zone of bistability, noise-induced transitions between attractors occur. In this zone, the lower attractor (stable equilibrium) coexists with the upper attractor (regular or chaotic). In spite of the fact that the initial state $x_{0}=0$ belongs to basin of attraction of the upper attractor, under the random disturbances with superthreshold intensity, random trajectories jump across the separatrix and localize near the lower stable equilibrium (Figures 2(c) and 2(d)). As a result, random states leave a basin of attraction of the upper attractor and localize near the stable equilibrium $M_{1}$ (see Figure 2(d)). Such transition can be interpreted as noiseinduced transformation from spiking regime to quiescence.

It is worth noting that such one-way transitions downwards occur only in the left interval $\left(\gamma_{1}, \gamma_{2}\right)$ of the bistability zone. For the right interval $\left(\gamma_{3}, \gamma_{4}\right)$, mutual transitions are observed. In Figure 3 for $\gamma=-2.8$, it is shown how separate deterministic attractors, equilibrium (lower) and chaotic (upper) (Figure 3(a)), combine into a single stochastic attractor (Figure 3(b)) under the noise of the intensity $\varepsilon=$ 0.15. Such transformation has an important interpretation from neuron activity point of view. Indeed, here noise generates a new bursting regime. Note that in the framework of the deterministic Rulkov model, more simple regimes of silence and spiking (periodic or chaotic) are observed only.

Stochastic phenomena, presented here on the base of direct numerical simulation, are studied in the next section with the help of the stochastic sensitivity function technique.

\section{SSF Analysis of Stochastic Bifurcations}

For the analysis of the dispersions of the random states, the approximations (see Appendix for details) based on the stochastic sensitivity functions technique are used. 


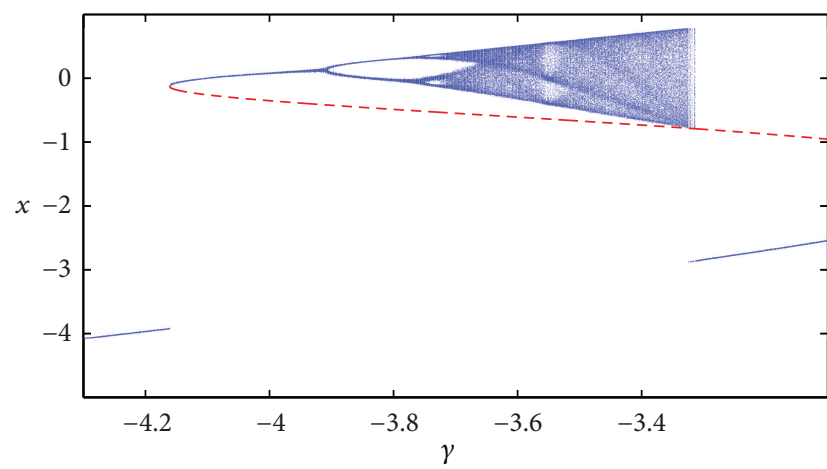

(a)

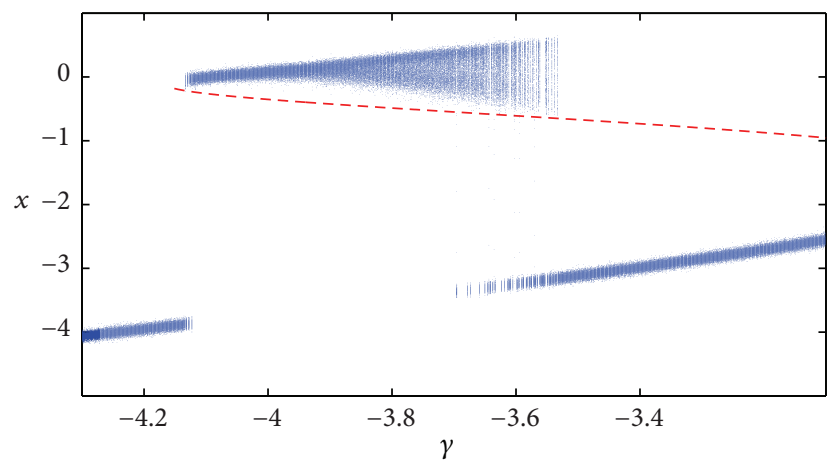

(c)

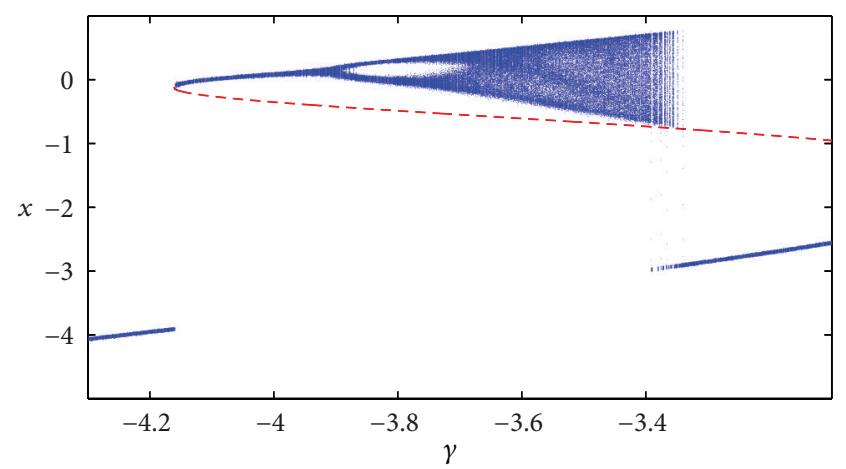

(b)

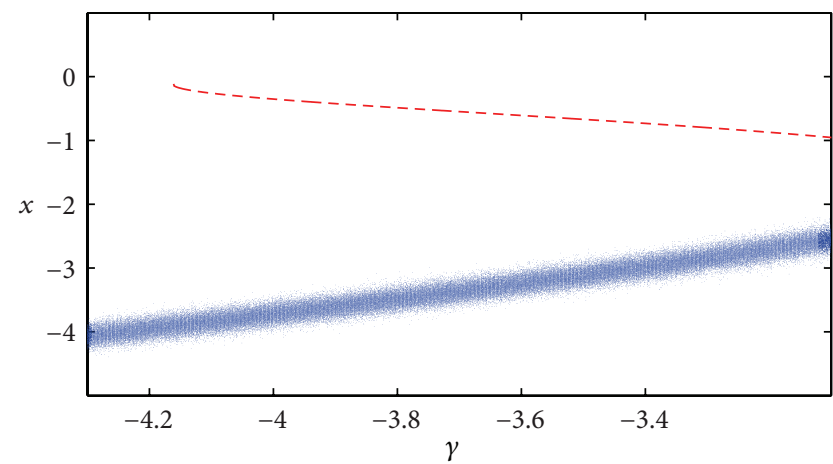

(d)

FIGURE 2: Random states of stochastic Rulkov model for (a) $\varepsilon=0.003$, (b) $\varepsilon=0.01$, (c) $\varepsilon=0.04$, and (d) $\varepsilon=0.1$.

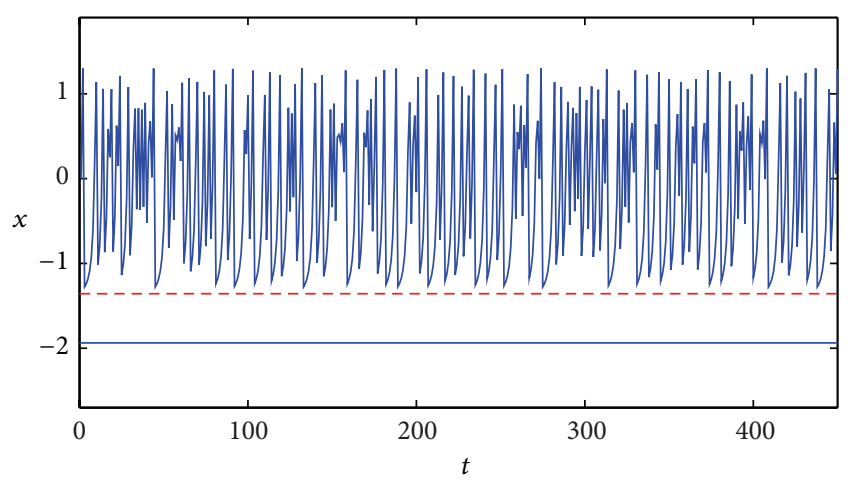

(a)

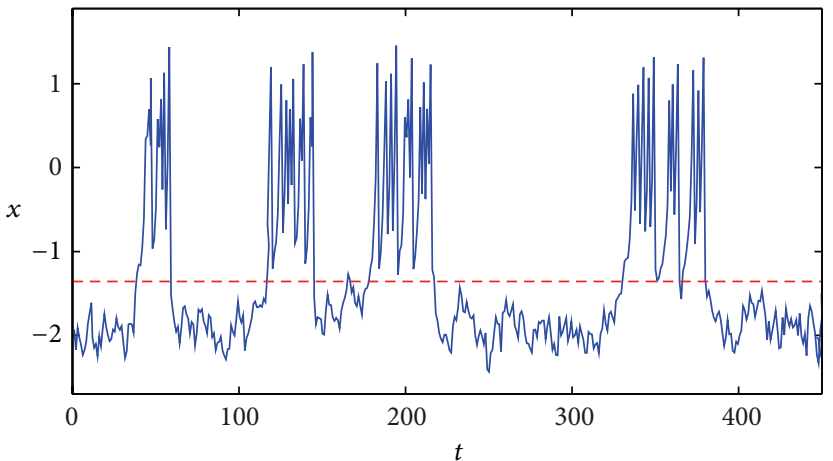

(b)

Figure 3: Time series for Rulkov model with $\gamma=-2.8$ : (a) $\varepsilon=0$, and (b) $\varepsilon=0.15$.

In Figure 4, the stochastic sensitivity for equilibria and cycles is shown. The stochastic sensitivity function $w(\mu)$ is plotted in Figure 4(a) for the equilibria $M_{1}(\mu)$ (blue color) and $M_{2}(\mu)$ (red color). As one can see, the stochastic sensitivity varies greatly at the edges of intervals of structural stability. Approaching the bifurcation points, this function unlimitedly increases.

The branches of the stochastic sensitivity function for the points of 2 cycles and 4 cycles are plotted in Figures 4(b) and 4(c). It can be seen that different points of the cycle have a different response on the random disturbances. Details of this difference are clearly observed with the help of the probability density function. In Figure 5, plots of the probability density function are shown for 2 cycles of system (1) with $\gamma=-3.9$ and three values of the noise intensity: $\varepsilon=0.003$ (red), $\varepsilon=0.007$ (green), and $\varepsilon=0.02$ (blue). Curves in Figure 5(a) were obtained by direct numerical simulation. In Figure 5(b), these curves were calculated by the formula (A.17) using the stochastic sensitivity function. A difference in the stochastic sensitivity of 2-cycle states implies the difference in the height and width of the peaks of the probability.

Consider a variation of the form of these peaks under increasing noise. For weak noise $(\varepsilon=0.003)$, a curve of the probability density function $\rho(x)$ has two narrow peaks. As 


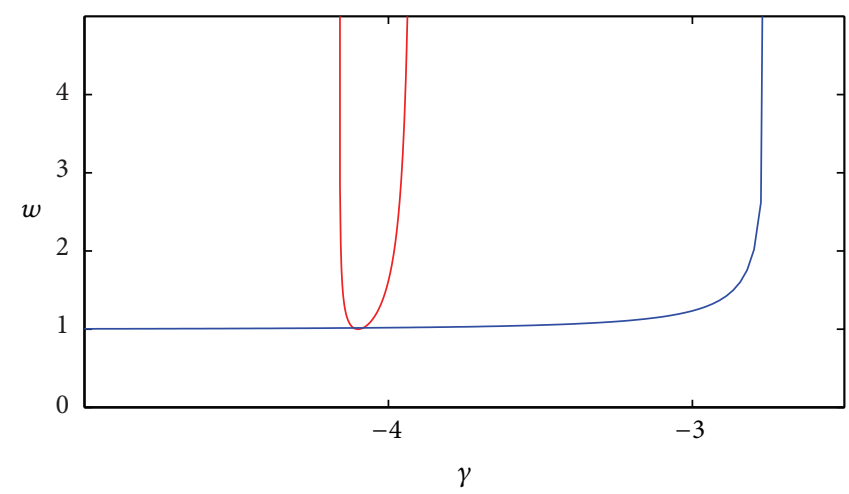

(a)

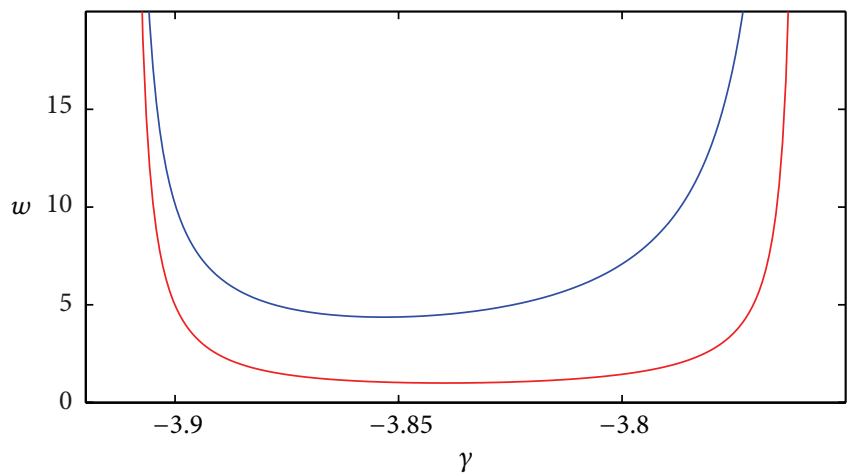

(b)

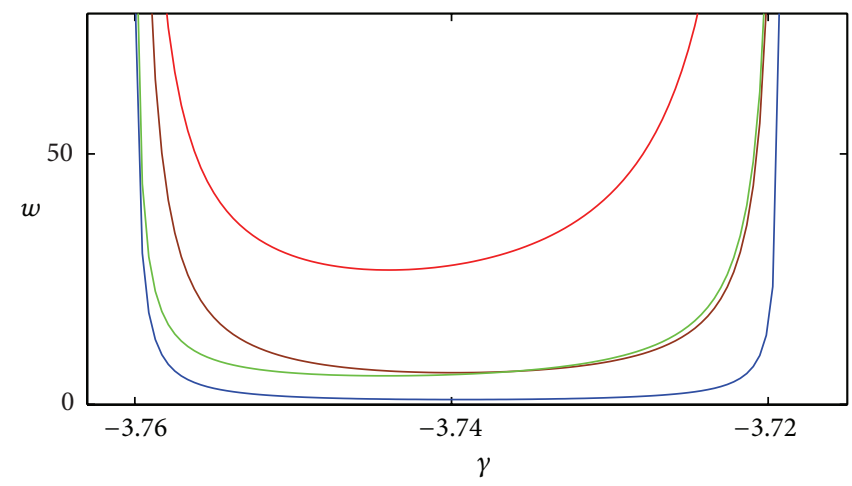

(c)

FIgURE 4: Stochastic sensitivity functions: (a) for equilibria $M_{1}, M_{2}$, (b) for 2 cycles, and (c) for 4 cycles.

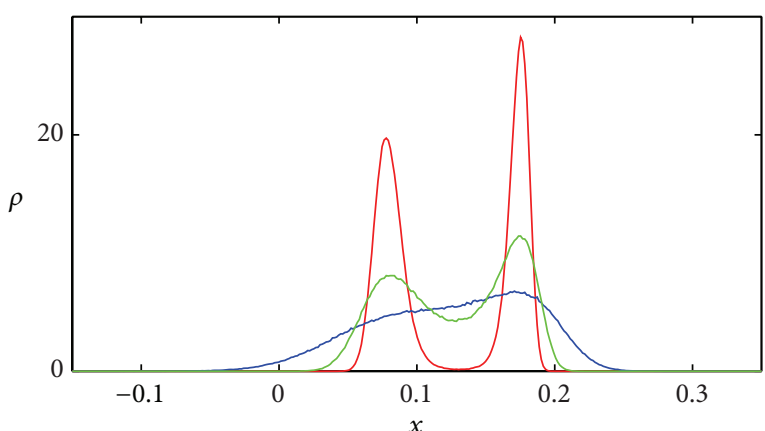

(a)

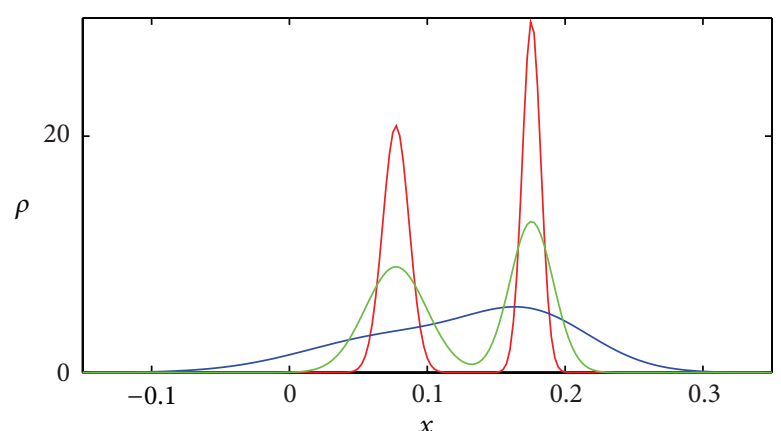

(b)

Figure 5: Backward stochastic bifurcation for $\gamma=-3$.9. Probability density functions for $\varepsilon=0.003$ (red), $\varepsilon=0.007$ (green), and $\varepsilon=0.02$ (blue).

the noise intensity increases, a process of merging of these peaks is observed. As one can see, a local minimum of $\rho(x)$ increases but the general graphic shape remains bimodal and a number of local extreme points equals three. For some threshold value $\varepsilon$, a qualitative change of the shape of $\rho(x)$ occurs. A form of this curve becomes unimodal. Two separate peaks transform into one and the only maximum remains, and stochastic 2 cycles look like a stochastic equilibrium. Such transformation of $\rho(x)$ from bimodal to unimodal form can be specified as a backward stochastic bifurcation. Similar transformation is observed for 4 cycles too. In Figure 6, it is shown how the backward stochastic bifurcation " 4 cycles $\rightarrow$
2 cycles" occurs. Plots in Figure 6(a) were extracted from the extensive direct numerical simulations and, in Figure 6(b), were found with the help of the analytical approximation (A.17).

It is worth noting that SSF technique allows us to analyze parametrically backward stochastic bifurcations for the multiple cycles. Such analysis is important for the understanding of the probabilistic mechanism of the stochastic deformation of the modality for the spiking regime.

In what follows, we show how this technique can be used for the study of noise-induced bursting. 


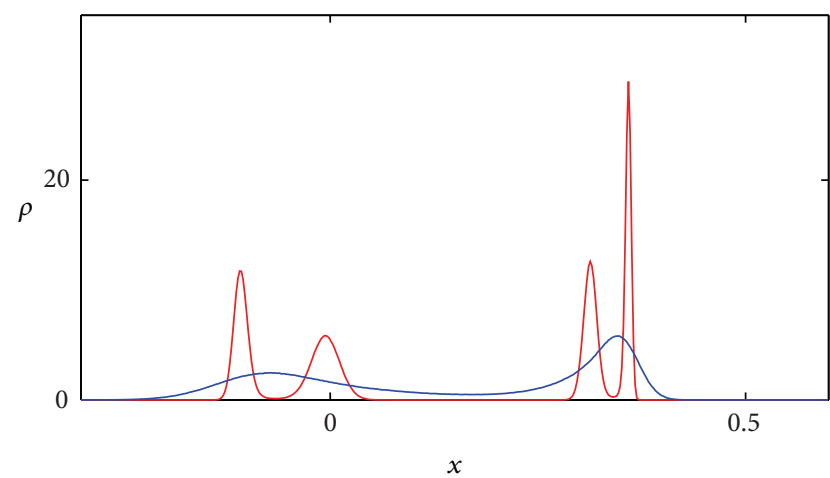

(a)

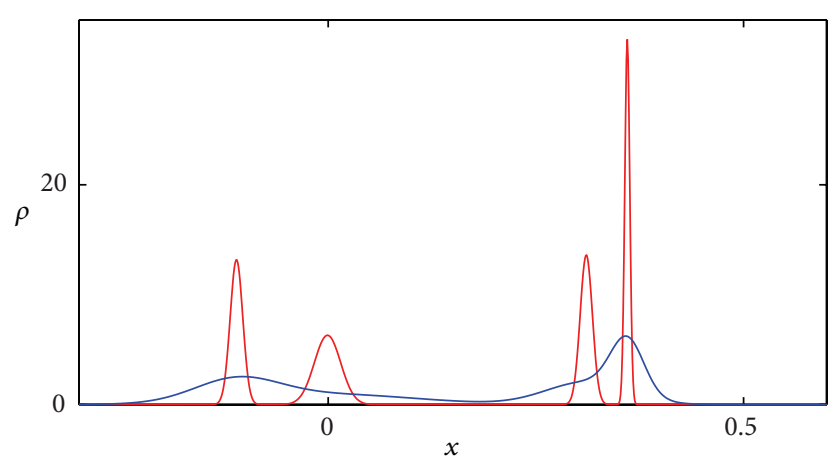

(b)

FIGURE 6: Backward stochastic bifurcation for $\gamma=-3.74$. Probability density functions for $\varepsilon=0.003$ (red) and $\varepsilon=0.02$ (blue).

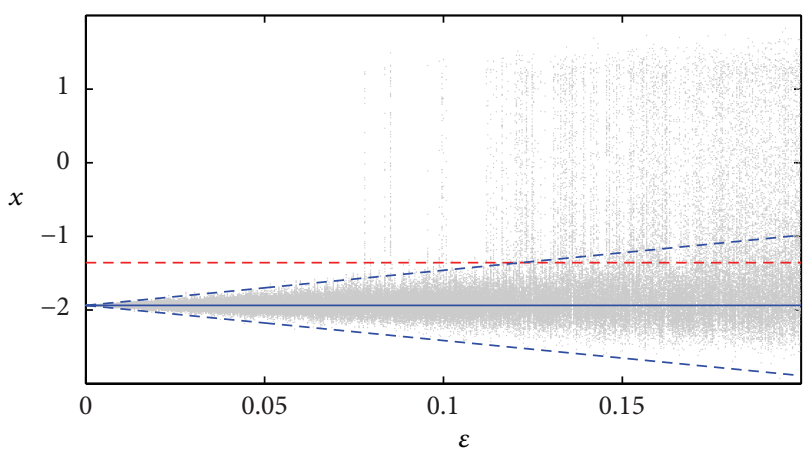

(a)

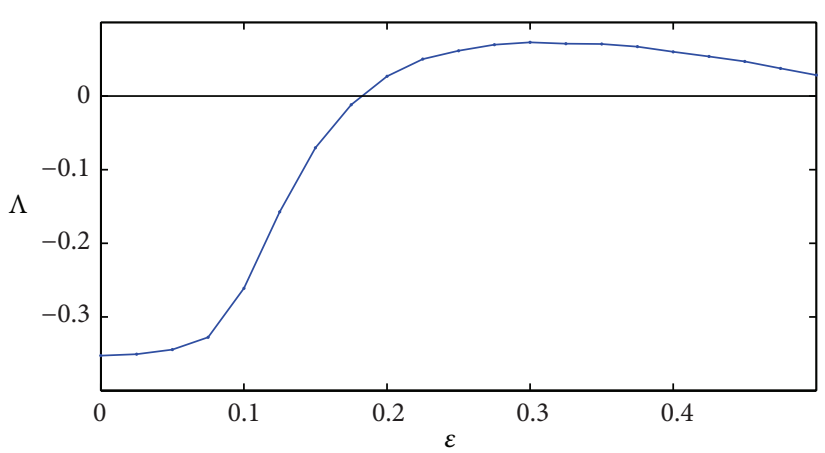

(b)

FIGURE 7: Noise-induced bursting (a) and noise-induced chaotization (b) for $\gamma=-2.8$.

\section{Noise-Induced Bursting and Transition to Chaos}

Consider Figure 3 again. In Figure 3(a), two separated attractors of the deterministic model (stable equilibrium and chaotic attractor) present two coexisting regimes of neuronal activity (quiescence and spiking). In Figure 3(b), a new wellmixed joined stochastic attractor models the bursting regime in system (1) for the noise intensity $\varepsilon=0.15$. Now, consider details of such transformation.

In Figure 7, random states (grey color) of Rulkov model with $\gamma=-2.8$ around the stable equilibrium (blue solid line) are plotted for the noise intensity $0 \leq \varepsilon \leq 0.2$.

For small $\varepsilon$, a linear growth of the dispersion is observed. It can be seen that there exists some transient zone of noise intensity values where the dispersion of random states abruptly grows. In this zone, random solutions of system (1) with high probability can cross the separatrix (unstable equilibrium, red line) and form bursting oscillations. Using SSF technique, we can estimate a position of this transient zone.

Boundaries of the confidence intervals around the stable equilibrium $M_{1}$ have been found by (A.8) and presented in Figure 7 by dashed lines. A point of the intersection of the upper boundary with the separatrix allows us to approximate a location of the transient zone of the onset of the bursting. As one can see, our theoretical method of the localization of the transient zone where the bursting oscillations appear is in a good agreement with results of the direct numerical simulations.

Consider now how these qualitative changes in the stochastic dynamics of the Rulkov model are connected with the changes in the largest Lyapunov exponent $\Lambda$. The largest Lyapunov exponent is a generally used quantitative characteristics for both deterministic and stochastic systems. The change of the sign of the largest Lyapunov exponent is a criterion of the transition from order to chaos [11, 20-22].

In Figure 7(b), a curve of the function $\Lambda(\varepsilon)$ is plotted for the Rulkov model with $\gamma=-2.8$. As noise intensity increases, a transition from the small-amplitude oscillations to largeamplitude bursts is accompanied by the change of the sign of $\Lambda$ from negative to positive. Such change of the sign of $\Lambda$ can be explained by the fact that the stochastic system spends a lot of time near the unstable equilibrium $M_{3}$ where the divergence dominates. In the transient zone, the fastest growth of $\Lambda(\varepsilon)$ is observed.

So, the noise-induced bursting in the Rulkov model is accompanied by the transition from order to chaos. 


\section{Appendix}

\section{A. SSF Technique}

Consider a nonlinear stochastic discrete-time system:

$$
x_{t+1}=f\left(x_{t}\right)+\varepsilon \sigma\left(x_{t}\right) \xi_{t},
$$

where $\xi_{t}$ is uncorrelated Gaussian random process with parameters $\mathrm{E} \xi_{t}=0, \mathrm{E} \xi_{t}^{2}=1$, and $\varepsilon$ is a noise intensity.

For the approximation of probabilistic distribution of random states around deterministic attractors (equilibria or cycles), the stochastic sensitivity functions technique can be used.

A.1. Stochastic Sensitivity of Equilibria. It is supposed that system (A.1) for $\varepsilon=0$ has an exponentially stable equilibrium $x_{t} \equiv \bar{x}$.

Let $x_{t}^{\varepsilon}$ be a solution of system (A.1) with the initial condition $x_{0}^{\varepsilon}=\bar{x}+\varepsilon v_{0}$. The variable

$$
z_{t}=\lim _{\varepsilon \rightarrow 0} \frac{x_{t}^{\varepsilon}-\bar{x}}{\varepsilon}
$$

characterizes the sensitivity of the equilibrium $\bar{x}$ both to initial and random disturbances. For the sequence $z_{t}$, it holds that

$$
z_{t+1}=a z_{t}+b \xi_{t}, \quad a=f^{\prime}(\bar{x}), b=\sigma(\bar{x}) .
$$

Dynamics of the second moments $v_{t}=\mathrm{E} z_{t}^{2}$ is governed by the equation

$$
v_{t+1}=a^{2} v_{t}+b^{2}
$$

For the exponentially stable equilibrium $\bar{x}$, it holds that $|a|<$ 1 and $v_{t}$ is stabilized for any $v_{0}$ :

$$
w=\lim _{t \rightarrow \infty} v_{t}=\frac{b^{2}}{1-a^{2}} .
$$

For small $\varepsilon$, a probabilistic distribution of $x_{t}^{\varepsilon}$ is stabilized too. It means that system (A.1) has a stationary distributed solution $\bar{x}_{t}^{\varepsilon}$ with probability density function $\rho(x, \varepsilon)$. This function has the following Gaussian approximation:

$$
\rho(x, \varepsilon) \approx \frac{1}{\varepsilon \sqrt{2 \pi w}} \exp \left(-\frac{(x-\bar{x})^{2}}{2 w \varepsilon^{2}}\right)
$$

with mean value $\bar{x}$ and dispersion $D=\varepsilon^{2} w$. The value $w$ links the intensity of stochastic input $\left(\varepsilon^{2}\right)$ with stochastic output $(D)$ in system (A.1) and characterizes a stochastic sensitivity of the equilibrium $\bar{x}$. For the stochastic sensitivity function $w$, the explicit formula can be written:

$$
w=\frac{\sigma^{2}(\bar{x})}{1-\left(f^{\prime}(\bar{x})\right)^{2}} .
$$

Values $w$ and $\varepsilon$ define the borders of the confidence interval $\left(x_{1}^{*}, x_{2}^{*}\right)$ :

$$
x_{1,2}^{*}=\bar{x} \pm k \varepsilon \sqrt{2 w} \text {. }
$$

Here the parameter $k$ is connected with fiducial probability $P$ by the formula $k=\operatorname{erf}^{-1}(P)$, where $\operatorname{erf}(x)=(2 / \sqrt{\pi}) \int_{0}^{x} e^{-t^{2}} d t$ is the error function. It means that random states of system (A.1) hit into this interval with the probability $P$. Confidence intervals characterize a spatial arrangement of random states of system (A.1) near the stable equilibrium $\bar{x}$.

A.2. Stochastic Sensitivity of Cycles. It is supposed that the deterministic system (A.1) $(\varepsilon=0)$ has $k$-cycle $\left\{\bar{x}_{1}, \ldots, \bar{x}_{k}\right\}$. Points of this cycle are connected by the equalities

$$
f\left(\bar{x}_{i}\right)=\bar{x}_{i+1} \quad(i=1, \ldots, k-1), \quad f\left(\bar{x}_{k}\right)=\bar{x}_{1} .
$$

The sequence $\bar{x}_{t}$ is defined for all $t$ due to the periodicity condition $\bar{x}_{t+k}=\bar{x}_{t}$. Let this cycle be exponentially stable. It means [23] that the following inequality holds:

$$
|a|<1, \quad a=a_{1} \cdot a_{2} \cdot \ldots \cdot a_{k}, a_{i}=f^{\prime}\left(\bar{x}_{i}\right) .
$$

For the asymptotics

$$
z_{t}=\lim _{\varepsilon \rightarrow 0} \frac{x_{t}^{\varepsilon}-\bar{x}_{t}}{\varepsilon}
$$

of the deviations of system (A.1) states $x_{t}^{\varepsilon}$ from the points $\bar{x}_{t}$, we have

$$
z_{t+1}=a_{t} z_{t}+b_{t} \xi_{t}
$$

where $a_{t}=f^{\prime}\left(\bar{x}_{t}\right), b_{t}=\sigma\left(\bar{x}_{t}\right)$.

A dynamics of the second moments $v_{t}=\mathrm{E} z_{t}^{2}$ is governed by the equation

$$
v_{t+1}=a_{t}^{2} v_{t}+b_{t}^{2}
$$

Due to inequality (A.10), the sequence $v_{t}$ is stabilized:

$$
\lim _{t \rightarrow \infty}\left(v_{t}-w_{t}\right)=0 \text {. }
$$

Here, $w_{t}$ is an unique $k$-periodic solution of (A.13).

For $w_{1}$, the explicit formula can be written:

$$
w_{1}=\frac{\left(b_{n}^{2}+b_{n-1}^{2} a_{n}^{2}+\cdots+b_{1}^{2} a_{2}^{2} \cdot \ldots \cdot a_{n}^{2}\right)}{\left(1-a^{2}\right)} .
$$

Other values $w_{2}, \ldots, w_{k}$ can be found recurrently:

$$
w_{i}=a_{i-1}^{2} w_{i-1}+b_{i-1}^{2} \quad(i=2, \ldots, k) .
$$

Values $w_{1}, \ldots, w_{k}$ of the $k$-periodic function $w_{t}$ characterize a response of the points $\bar{x}_{1}, \ldots, \bar{x}_{k}$ of the cycle to small random disturbances. The vector $w=\left(w_{1}, \ldots, w_{k}\right)^{\top}$ is called the stochastic sensitivity function of the cycle.

Probability density function $\rho(x, \varepsilon)$ of the stationary distributed random states of system (A.1) near points $\bar{x}_{1}, \ldots, \bar{x}_{k}$ of the cycle can be approximated as follows:

$$
\rho(x, \varepsilon) \approx \frac{1}{\varepsilon k \sqrt{2 \pi}} \sum_{i=1}^{k} \frac{1}{\sqrt{w_{i}}} \exp \left(-\frac{\left(x-\bar{x}_{i}\right)^{2}}{2 w_{i} \varepsilon^{2}}\right) .
$$


Stochastic sensitivity function (SSF) technique was elaborated for the analysis of randomly forced equilibria and limit cycles for both continuous [18] and discrete-time [17] systems. The SSF technique was successfully applied to the study of noise-induced intermittency with a transition to chaos [19] and stochastic bifurcations [24].

Confidence domains are sufficiently simple and evident geometrical models for the spatial description of random states of the stochastic system. The SSF technique enables to construct confidence intervals, ellipses, bands, and tori for the probabilistic analysis of various stochastic attractors. Constructive potentialities of the confidence regions method have been demonstrated in the analysis of stochastic phenomena for population [18] and neuronal [14] systems.

\section{Conflict of Interests}

The author declares that there is no conflict of interests regarding the publication of this paper.

\section{Acknowledgment}

This work was partially supported by the RFBR (14-01-00181).

\section{References}

[1] A. L. Hodgkin and A. F. Huxley, "A quantitative description of membrane current and its application to conduction and excitation in nerve," Journal of physiology, vol. 117, no. 4, pp. 500$544,1952$.

[2] E. M. Izhikevich, Dynamical Systems in Neuroscience: The Geometry of Excitability and Bursting, Computational Neuroscience, MIT Press, Cambridge, UK, 2007.

[3] B. Ibarz, J. M. Casado, and M. A. F. Sanjuán, "Map-based models in neuronal dynamics," Physics Reports, vol. 501, no. 1-2, pp. 174, 2011.

[4] M. Girardi-Schappo, M. H. R. Tragtenberg, and O. Kinouchi, "A brief history of excitable map-based neurons and neural networks," Journal of Neuroscience Methods, vol. 220, no. 2, pp. 116-130, 2013.

[5] N. F. Rulkov, "Regularization of synchronized chaotic bursts," Physical Review Letters, vol. 86, no. 1, pp. 183-186, 2001.

[6] S. Rajasekar, J. Used, A. Wagemakers, and M. A. F. Sanjuan, "Vibrational resonance in biological nonlinear maps," Communications in Nonlinear Science and Numerical Simulation, vol. 17, no. 8, pp. 3435-3445, 2012.

[7] C. Wang and H. Cao, "Parameter space of the Rulkov chaotic neuron model," Communications in Nonlinear Science and Numerical Simulation, vol. 19, no. 6, pp. 2060-2070, 2014.

[8] W. Horsthemke and R. Lefever, Noise-Induced Transitions, vol. 15 of Springer Series in Synergetics, Springer, Berlin, Germany, 1984.

[9] K. Matsumoto and I. Tsuda, "Noise-induced order," Journal of Statistical Physics, vol. 31, no. 1, pp. 87-106, 1983.

[10] F. Gassmann, "Noise-induced chaos-order transitions," Physical Review E, vol. 55, no. 3, pp. 2215-2221, 1997.

[11] J. B. Gao, S. K. Hwang, and J. M. Liu, "When can noise induce chaos?" Physical Review Letters, vol. 82, no. 6, pp. 1132-1135, 1999.
[12] N. Hritonenko, A. Rodkina, and Y. Yatsenko, "Stability analysis of stochastic Ricker population model," Discrete Dynamics in Nature and Society, vol. 2006, Article ID 64590, 13 pages, 2006.

[13] B. Lindner, J. García-Ojalvo, A. Neiman, and L. SchimanskyGeier, "Effects of noise in excitable systems," Physics Reports, vol. 392, no. 6, pp. 321-424, 2004.

[14] I. Bashkirtseva, A. B. Neiman, and L. Ryashko, "Stochastic sensitivity analysis of the noise-induced excitability in a model of a hair bundle," Physical Review E: Statistical, Nonlinear, and Soft Matter Physics, vol. 87, no. 5, Article ID 052711, 2013.

[15] C. Gardiner, Stochastic Methods: A Handbook for the Natural and Social Sciences, vol. 13 of Springer Series in Synergetics, 4th edition, 2009.

[16] A. Lasota and M. C. Mackey, Chaos, Fractals, and Noise: Stochastic Aspects of Dynamics, Springer, New York, NY, USA, 1994.

[17] I. Bashkirtseva, L. Ryashko, and I. Tsvetkov, "Sensitivity analysis of stochastic equi-libria and cycles for the discrete dynamic systems," Dynamics of Continuous, Discrete and Impulsive Systems Series A: Mathematical Analysis, vol. 17, no. 4, pp. 501-515, 2010.

[18] I. Bashkirtseva and L. Ryashko, "Sensitivity analysis of stochastic attractors and noise-induced transitions for population model with Allee effect," Chaos, vol. 21, no. 4, Article ID 047514, 2011.

[19] I. Bashkirtseva and L. Ryashko, "Stochastic sensitivity analysis of noise-induced intermittency and transition to chaos in one-dimensional discrete-time systems," Physica A: Statistical Mechanics and its Applications, vol. 392, no. 2, pp. 295-306, 2013.

[20] J. P. Crutchfield, J. D. Farmer, and B. A. Huberman, "Fluctuations and simple chaotic dynamics," Physics Reports, vol. 92, no. 2, pp. 45-82, 1982.

[21] H. G. Schuster, Deterministic Chaos, Physik-Verlag, Weinheim, Germany, 1984.

[22] O. Martin, "Lyapunov exponents of stochastic dynamical systems," Journal of Statistical Physics, vol. 41, no. 1-2, pp. 249-261, 1985.

[23] S. N. Elaydi, An Introduction to Difference Equations, Springer, Berlin, Germany, 2nd edition, 1999.

[24] I. Bashkirtseva, L. Ryashko, and P. Stikhin, "Noise-induced backward bifurcations of stochastic 3D-cycles," Fluctuation and Noise Letters, vol. 9, no. 1, pp. 89-106, 2010. 


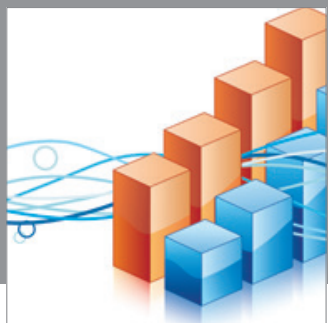

Advances in

Operations Research

mansans

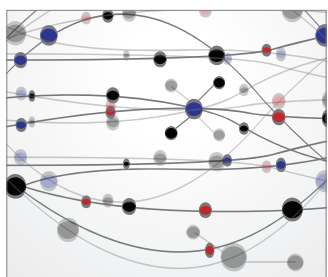

The Scientific World Journal
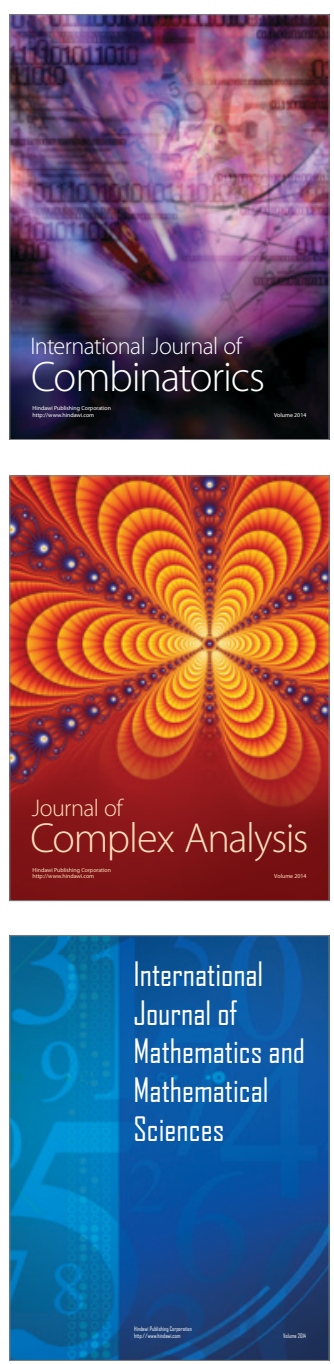
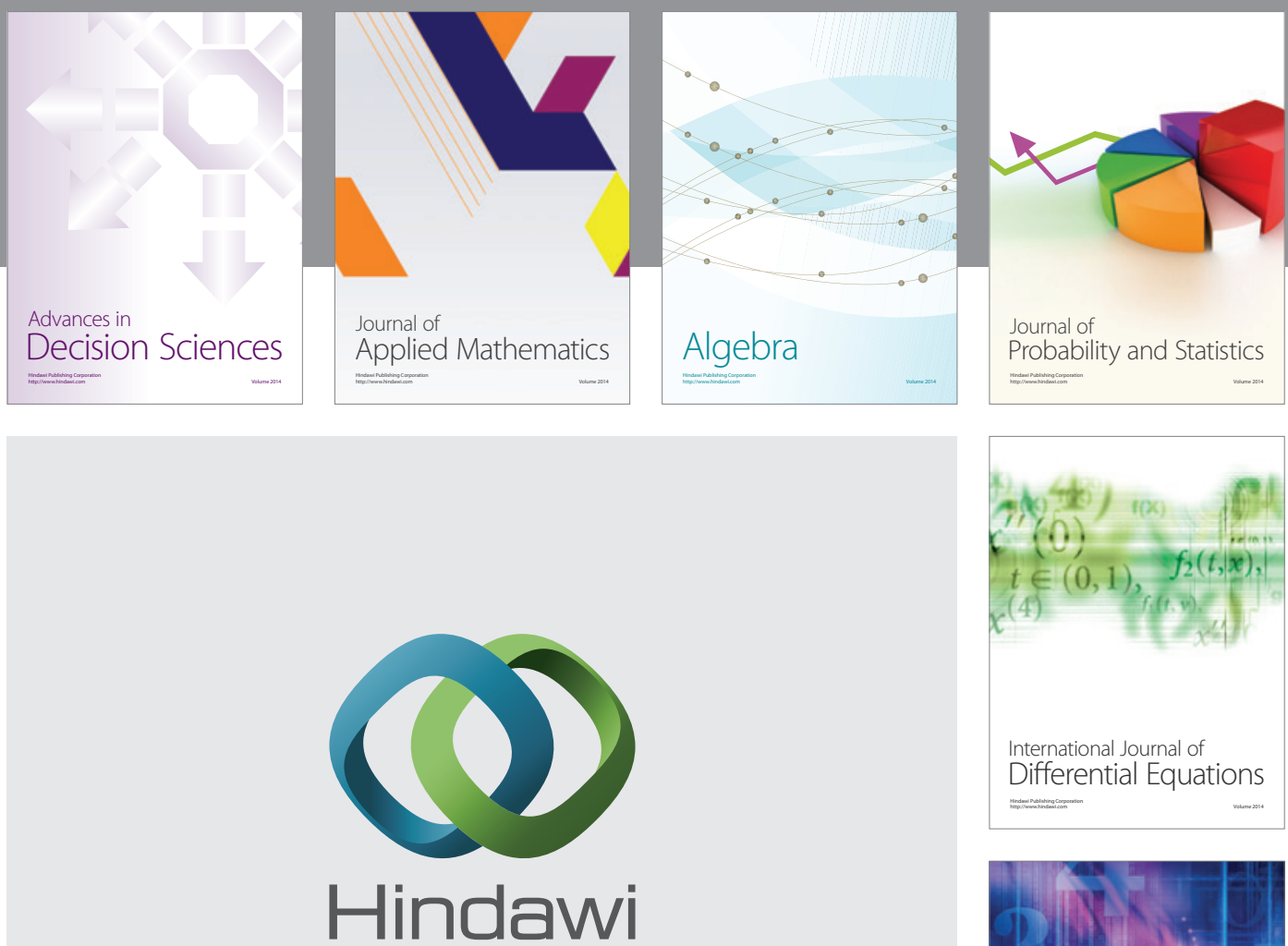

Submit your manuscripts at http://www.hindawi.com
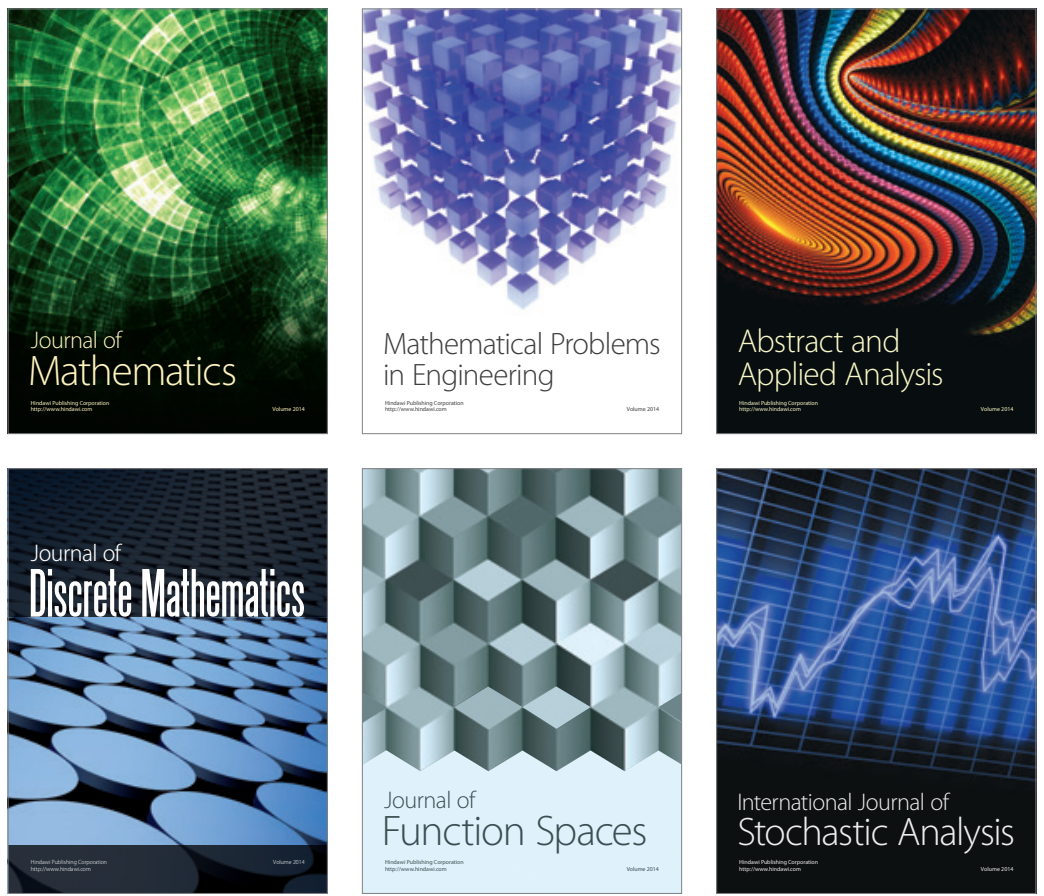

Journal of

Function Spaces

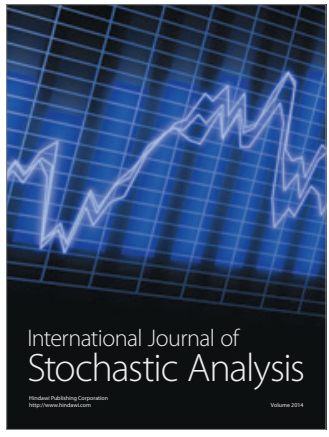

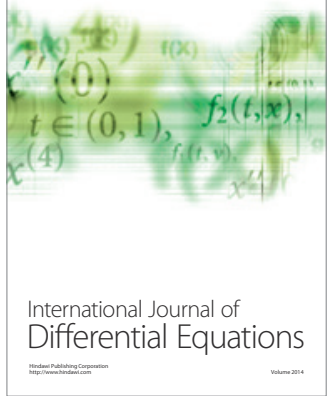
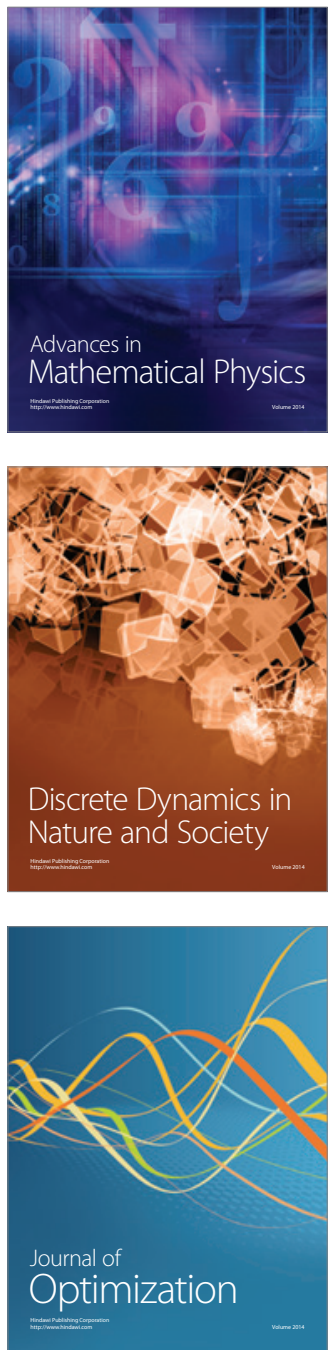